\title{
A Multi-Agent Approach for Performance Based Architecture: Design exploring geometry, user, and environmental agencies in façades
}

\author{
Authors: David J Gerber ${ }^{2}$, Evangelos Pantazis², Alan Wang1 \\ ${ }^{1}$ School of Architecture, University of Southern California, Watt Hall 204, Los Angeles, CA 90089, USA \\ 2Sonny Astani Department of Civil and Environmental Engineering, University of Southern California, 3620 S. \\ Vermont Avenue, KAP 217, Los Angeles, CA 90089, United States
}

\begin{abstract}
:
This paper presents research on the use of multi-agent systems (MAS) in architectural design with the goal of both improving and partially automating the design process as well as the design outcomes in terms of geometry and environmental performance. The work is based upon research on agent based modelling and simulation techniques (ABMS), and the combination of analytical and optimization methods with geometric modelling and user centered design. The motivation is to render more accessible the exploration of the generative aspect of bottom up design techniques when coupled with optimization and performance criteria. The objective of the work is to develop and test a design methodology, which enables designers to explore larger sets of informed solutions by coupling design requirements and generative design techniques with validated simulations (i.e., environmental analysis) based partly on the inclusion of user light preferences early in the design process. The development of the proposed methodology and the implementation of the design system for a building façade are described, while the robustness and validity of the framework is initially tested by applying it to the generation and optimization of façade panel configurations of an office building. The results show that the MAS for design system can generate unique design configurations that perform environmentally (daylight factor analysis) better than a façade with normative façade shading.
\end{abstract}

Keywords: Multi Agent System; Design Automation; Generative Design Systems; Agent Based Modeling; Performance Based Design

\section{INTRODUCTION}

With the increased access to computational design tools and the consequent rapid evolution of algorithmic design methods [49,73], associative parametric modeling [17,39], and multi-disciplinary design optimization [19], design teams have been provided with a new set of design exploration possibilities. As a result, architecture is now experiencing a proliferation of approaches and techniques to generate, rationalize, and optimize design processes and outcomes. In the past decade, simulation has played a more significant role in the design practice for gaining insight and evaluating different performance criteria, including risk, cost, energy efficiency, structural efficiency, lighting, and social utility $[7,13,80]$. In architectural and urban design discourse, there exists a burgeoning interest in agent based modeling and simulation (ABMS) and in non-linear systems as a means to not only improve the design process but also to improve design exploration through adaptation and emergence, facilitating the production of higher performing design outcomes without reducing geometric intricacy. Given these developments, an increasing number of building designs incorporate geometrically complex freeform building envelopes and structures [53]. Modeling of non-Euclidean geometries and exchanges of data between expert disciplines have facilitated the need for advancing active synchronous collaboration, more rapid exploration of design alternatives, and greater management of the complexity of design problems. In some cases the design modeling has been inclusive of environmental settings and information (e.g., daylight factor analysis, heat gain), user centered (e.g., end-user preferences and design feedback) and structural (e.g. stress analysis, force/ flow distribution) feedback loops to name a few [18,23,35,39]. Although, there has been a significant interest in the performance based design and the development of integrated workflows for environmental factors [8], there remains a number of critical opportunities to integrate further the ability to design explore in an empirically informed fashion where design creativity is enhanced by bottom up exploration methods and engineering based optimizations. In this paper, we point to the lack of contribute to the development of integrated and automated digital workflows during the design 
process and we introduce a design methodology which; (1) can combine generative design techniques with analytical data and user collected data as optimizing agencies; (2) is extensible; and (3) adaptable to different design variables, design topologies, and types of analysis, such as environmental and/or structural analysis. We utilize multi-agent systems (MAS), where a combination of multiple agent types is used.

By considering the computer as an active collaborator of the designer, one of our research goals is to enable design teams to quickly generate, measure, and optimize complex geometries across a set of complexly coupled design objectives and in this iteration of the MAS, specifically, environmental performance analyses. The work is also motivated to address a notable gap in architecture's application of emergent systems and generative agent strategies, namely that the majority of the precedent work in the literature is based on a single type of approach, that of the swarm or boid. The work conjectures that through the development of a MAS, design teams can intuitively explore a larger design solution space by integrating analysis tools with user related data in order to inform and improve an automated design process $[41,60,65,74]$. The research developed presents a generative design system that offers design alternatives that go beyond the geometry-only related objectives but rather and uniquely, are informed by environmental analysis and user preferences i.e., performance criteria and objectives. The research goals are manifold including: (1) a desire to bring to architecture the state of the art in non-linear and emergent design systems; (2) enable the realization of intricate and formally complex designs, measured for their empirical performance across multiple objectives; and (3), to measure the benefits of a MAS approach for aiding design teams to design with complexity and with greater certainty in the performance criteria discussed subsequently. The overall goal is to generate complex and freeform architecture that is equally outperforming manual and deterministically limited solution spaces across multiple performance objectives, highly relevant to contemporary quotidian design practices [38,39,59]. It should be noted the demonstration design use case, a geometrically complex façade, is purposefully idiosyncratic and intricate, however the system is conceived as applicable to more normative geometric systems, nevertheless we use the complex as a purposeful base case for testing the system but also for demonstrating how complex can be found to out perform the simple. The proposed design methodology's outcomes contribute to the improvement of the design process by reducing the design cycle latency through producing more valid design alternatives from which a design decision can be made more rapidly as well as produces more optimal design variants than those produced in a purely manual, deterministic or non generative approach.

Specifically, in this paper, we apply, test and calibrate the proposed MAS design technique, as well as illustrate a design case study for measuring the benefits and limitations of such an approach in an architectural context. In order to provide a better understanding of the MAS design technique introduced in this paper, an experimental case study that integrates end-users lighting preferences with environmental analyses early in the design process of a building envelope with goals to increase daylight availability in the interior space and improve the end-user's comfort is presented. The experimental case study includes the development and implementation of a MAS for the generation of design alternatives of a non-structural building component (i.e., façade panels), which is based on combining environmental simulation and analysis and user preferences constrained by geometric and fabrication logics and limits. The system is run based on collected data from participants in a virtual office space. The results include a set of geometrically intricate design alternatives, which account for both improved energy efficiency and user preference targets.

The paper is structured through six sections. In section 2 we present a literature survey on the use of MAS in Architecture Engineering and Construction (AEC) and related domains, and our agent definitions. In section 3 the design problem, research objectives, methodology and the implementation of our system for a specific experimental design case are described. In section 4 the results and analysis of the experimental design are presented. In section 5 limitations of the research to date and future work are outlined. Lastly, in section 6 a summary of contributions and conclusions of the research are discussed. 


\section{Agent Based Modeling and Multi-Agent Systems}

Research has shown that the complexity and uncertainty, encountered in design problems, can be more effectively addressed with distributed computation and artificial intelligence [27,43,79]. However, given the nature of design problems as 'ill structured', designers must engage in defining abstractions in order to design explore and optimize computationally $[56,64]$. The capacity of distributed systems, in this case of a MAS, to abstractly model requirements as agent goals and to adapt to local conditions, has rendered them appropriate for solving a large class of real world problems in a number of domains, including software engineering, financial markets, security and game theory $[71,75,78]$ to name a few. MAS are also related to Complex Adaptive Systems (CAS), which are characterized by their ability to self-organize and dynamically reorganize their components in different ways and across multiple scales [31]. This process allows the agents to negotiate, survive, and adapt within their environments [43], and incorporates evolutionary and generative mechanisms found in nature and the development of mathematical models that simulate physical processes [10]. There exist a number of properties, such as aggregation, nonlinearity, flows, diversity, and mechanisms, such as planning and tagging of internal models as building blocks that are common to MAS and serve as a reference for designing and developing agent-based models that can be synthesized to form a MAS $[28,43,70,75]$. Due to their modularity, MAS are considered applicable for producing portable, extensible, and transferable algorithms, with better integrated development environments and more applications [43,69].

It should be noted that application of MAS in the AEC industry has been less pervasive. Beetz classifies MAS in AEC under three domains of design generation, namely: knowledge capturing and pattern recognition; simulation and performance of building designs; and collaborative environments [9]. In the fields of engineering and construction, researchers have been exploring the applicability of MAS from different perspectives, such as for collaborative design, construction scheduling and structural optimization to name a few [5,9,30,41]. Agent based simulations have been used in digital fabrication and building construction for their capability to abstract, adapt and simplify real time complexities into simple basic rules [58]. Additionally, there has been impactful research in developing MAS for autonomous collective construction both at the level of algorithms but also at the level of hardware [76,77]. In the field of architecture and computational design, the focus of research so far has been mostly on design generation (form and aesthetic) and simulations [22,61]. Approaches to design generation can be classified as linear and non-linear based on algorithms that operate, either in top-down or bottom up fashions [21,24,63]. Many have argued that top down approaches offer control though not enough design flexibility as they operate on fixed design topologies that are sequentially decomposed [67]. On the other hand, bottom up algorithms can be challenging to apply for design purposes and often exhibit a lack of control in the design outcome [68]. We have found in the literature, a majority of the research in bottom up design approach for architecture has focused on the generative aspects, which have predominantly implemented swarm or boid algorithms $[6,12,15,29,41,44]$. Snooks argues "Swarm intelligence" enables the encoding of design requirements either into agent behaviors of different populations that belong to interrelated sub systems or within a population with adjustable or differentiated behaviors of one system [41,42]. The distributed nature of agent based models enables the mutual negotiation of relationships between different design parameters, such as program and form or structure and ornament [65]. Whereas, Menges uses swarmbased agent models in order to establish communication, across different design environments (architectural design, structural design) and for different hierarchical levels (global geometry, material structure) and thus allowing for the uninterrupted flow of information from input parameters into multiple design constraints [47,51]. However, according to Anumba et. al, the encoding of the design requirements (i.e. building design requirements) into agent behaviors and the definition of an agent upon the decomposition of a given design problem, is most often highly complex and consequently, hard to achieve [4,5]. Therefore, although there is considerable development and utilization of distributed/bottom up models in computer science and other fields of engineering, the direct implementation of MAS achievements in AEC is not as easy due to the gap in the degree of formalization of problem requirements during the building design process [10]. Soibelman et al. implement an agent based reasoning model to enable designers to more rapidly explore conceptual 
structural designs for tall buildings. In their approach a MAS system (M-RAM) provides the designer with adapted solutions for evaluation. The solutions are generated by a distributed multi-constraint reasoning mechanism [66]. Focusing more on pattern recognition and the representational aspect of design problems, Achten has proposed a MAS framework for graphic unit recognition in technical drawings. This approach suggests that singular agents may specialize in graphic-unit recognition and MAS can address problems of ambiguity through negotiation mechanisms [2]. In the field of building performance evaluation and control, Dijkstra and Timmermans created a custom platform, AMANDA, to simulate pedestrian flows in urban environments [14]. Meissner has used MAS and agent based simulations for the support and integration of fire protection engineering into the planning process [46], while Klein et al. have used MAS in combination with Markov Decision Problems (MDP) in order to develop alternative building management and control systems in relation to occupant habits and preferences [37].

Even though there has been a number of examples, where agent based models have been used to simulate user behavior, such as circulation patterns, the AEC industry has done little to adapt techniques to accurately incorporate end-user's behavioral and performance information during the design phase of buildings [33,63]. Studies have shown that if buildings are designed according to their user needs, behavior and preferences, there is potential to reduce the total energy consumption in the building during its operation phase [16]. Additionally, research within other domains and industries, such as security [1,72], economics [50], and game theory [32], has shown that user-centered designs could significantly increase the efficiency of systems. A common approach to incorporate user-related information during the design phase is through the use of behavioral models to simulate users' movements, interactions, and responses within the designed environment, which are also used to estimate building's energy consumption more accurately based on occupants' possible interactions, comfort levels, and preferences during the design phase [34,36]. Although such simulations have been promising and provide a more user-centered analysis of a building's operations, in many cases due to complexities of human behavior (e.g., preferences, personalities, etc.), they do not provide accurate and realistic representation of actual occupants' behavior during the operation phase; therefore, in some cases the building could be less energy efficient and not accommodate the occupants' needs [11]. Therefore, there is a lack of a holistic approach, which has the capacity to integrate generative design rules with (1) user related information (i.e. preferences), (2) multiple analyses and performance criteria such as environmental and lighting analysis, (3) building and material constraints, and (4) performance optimization functions.

In architectural design, the vast majority of the research has relied upon a simple agent-based swarm or boid model, generally focused on the generation of geometry centric and aesthetically intricate design outcomes. Thus we conclude, inadequate research has been done in combining generative processes with analytical processes and user related data in a distributed fashion, as well as, in adapting and utilizing more sophisticated agent based algorithms such as Markov Decision Problems and team formation models. We also note there are two critical impediments to the furtherance of MAS in general and specifically for architecture; (1), that there exists a lack of methodology to enable (software) designers to clearly specify and model their applications as MAS, and (2), the lack of widely available MAS toolkits that support designers to effectively explore larger and more informed solution spaces. In response to a perceived gap and over dependence on the aesthetic an un-analytical implementations of the swarm, we collaborated on the development of a novel approach for a collaborative MAS, which combines alternative agent models (social choice) with number theory, where the MAS is applied to optimize efficient building design [45]. This approach presents teams of uniform and diverse agent populations with different design and performance goals. The developed system aggregates the agents' opinions, which relate to a predefined range of design requirements, in order to provide designers with a larger number of pareto optimal design solutions [40].

To provide clarity, an "agent" in our work is denoted as a software-based computer system that shares the following properties: (1) an agent exists within an environment and responds to it while interacting with other agents and is therefore 'situated' in that its behavior is based on the current state of its interactions with both the population of the agents as well as with the environment; (2) an agent 
may have explicit objectives that condition its behavior and is directly related to specific performance criteria in which the goals are not solely targeted to maximize effectiveness but are used to assess and improve the decision making process; (3) an agent can adapt and change its behavior based on analytical data or its own evolution and interaction history; in this case, individual adaptation requires agents to have memory to keep track of their actions usually in the form of a dynamic agent parameter, and therefore, (4) an agent has resource parameters that indicate its current stock of one or more resources (energy, material, information) [43].

\section{RESEARCH METHODOLOGY}

The specific hypothesis of this research states that for the façade of an office building environment a bottom up design approach, which incorporates environmental performance analysis coupled with user preferences for daylight levels, will provide a larger set of design alternatives that perform better than those conventionally generated through non MAS based design methods. The proposed system consists of a series of negotiating subsystems i.e. "agencies," each focusing on their individual design objectives, including end-user preferences in conjunction with environmental performance parameters and basic fabrication constraints. The success and/or failure of the experiment is measured against a normative benchmark condition with regular openings and cross-compared against starting design and final design options. The parameters in the research include, (1) environmental performance criteria as they relate to established standards and user preferences, (2) quantity of equivalent and higher performing design solutions, and (3) generative capacity of the system to create unique and complex outcomes, which improve upon each iteration. Here, we formulate a corollary between the design across multiple objectives and overall performance of the system, and utilize design evolution and emergence to aid in that success. By doing so, the research begins to prove out the argument that bottom up MAS methodologies and technologies should be used not only for aesthetics but more so for design inclusive of performance objectives. Through the work, we specifically attempt to prove that our MAS emergent design technique can lead to an increased set of higher performing solutions than those achievable through purely linear, manual, and or deterministic parametric modeling. In that regard we introduce, test and validate a MAS based design methodology and technology for its ability to simultaneously optimize across complexly coupled objectives and its ability to provide design teams with higher performing design solutions while maintaining geometric intricacy and design intent. Another important contribution is to introduce, test and validate more sophisticated agent behaviors that go beyond the current architectural standard agent models such as flocking, swarming boids which include probabilities and communication mechanisms (with other agents) as a means to improve upon them. The problem is de-composed into sub-domains that include: generation of design solutions, simulation and analysis of their environmental performance, and evaluations based on user collected data and performance metrics (Figure 1). The overall purpose is to improve the generative and design decision making process based on analytical results and user collected data [45].

\section{DESIGN APPROACH}

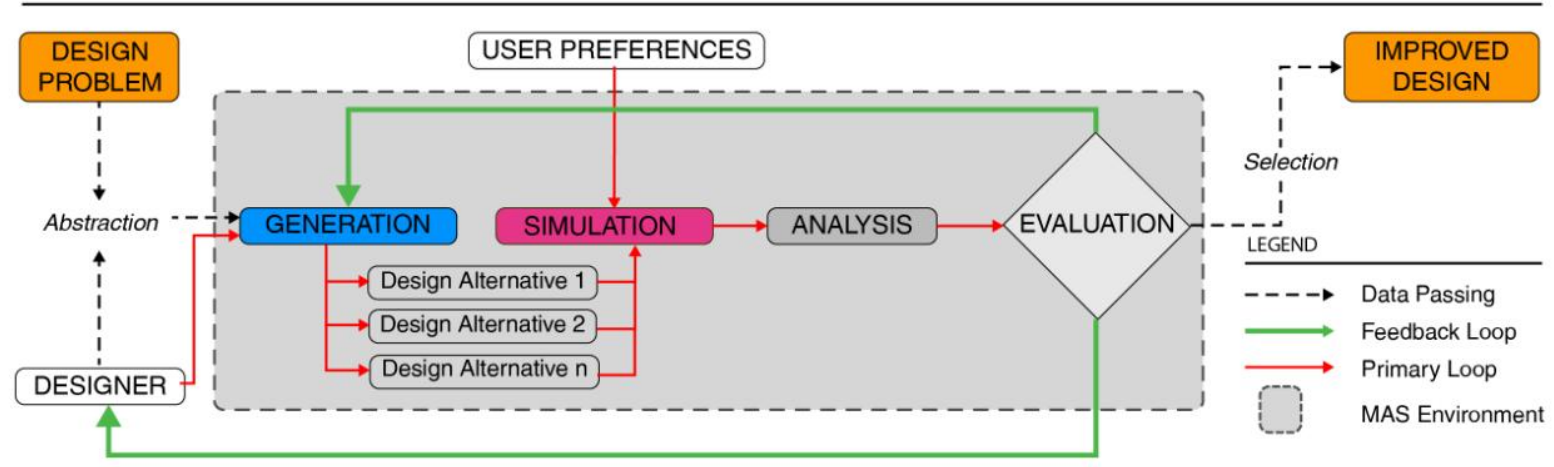

Figure 1: Diagram illustrating the overall Multi-Agent Systems for design approach including design problem, designer interaction, results, and feedback loops and the MAS environment decomposition of the system into the subdomains of design generation, simulation, analysis and evaluation. 


\subsection{Experimental Design Case: Light Diffusing Façade Panel}

Lighting accounts for $20-25 \%$ of the total electrical use in buildings, and more specifically $30-50 \%$ in commercial buildings [3,52]. Building façades in large part determine the amount of available direct and indirect natural lighting in the building $[8,55]$. Façades are also among the most complex architectural systems, combining aesthetic, structural, environmental, and construction concerns [8]. Therefore, a focus on the architectural façade and the experimental design investigates how by combining environmental analysis data, specifically solar radiation and luminance, with user preferences for light intensity within the office environment, our MAS can improve the design and performance of the building façade. The experiment is divided into two sections: (1) the development of a generative agent based façade paneling system that is optimized based on environmental performance analysis, and (2) the adaptation of designs based on specific user preferences, which are collected and profiled, based on how they relate to lighting conditions within the office space. $\mathrm{We}$ used the collected light preferences as an input and means to formulate goals that drive our system towards optimality. The lighting preference data, was collected from 89 participants. Details about the collection of user preferences can be found in [26]. For simplicity at this stage, we used the preferences of a single group of users, which preferred more natural light (23\% of all participants). Although this group does not reflect the most preferred lighting setting among our sample, we selected this group in order to test the ability of our system to generate more energy efficient solutions as one of the initial validation experiments. We define the increase of natural light, its distribution inside the office and the satisfaction of user preferences as design requirements. In order to evaluate our MAS design methodology, we applied it on a generic office space located in Los Angeles California. The office space is located on the 4th floor of a 30-year-old facing south commercial building. The façade has two openings and a glazing ratio of approximately 50\% (Figure 2A-B). The office space is analyzed annually in simulation (Figure 2D). Yet, the key dates, here the extremes, are selected by the designer and compared for a specific period of the day, which correspond to the main operational hours of a typical commercial building ( 9 a.m. - 6 p.m.).

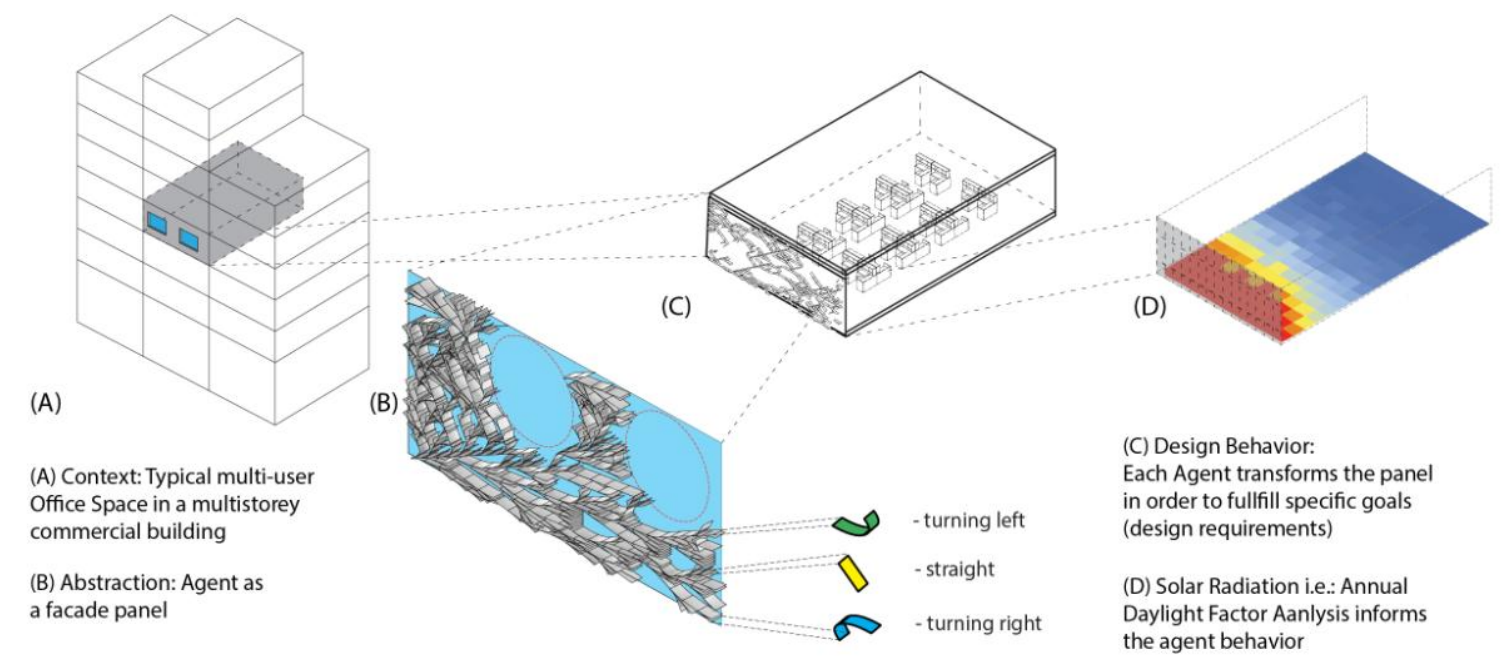

Figure 2: Diagram describing, (A) the specific design context of the building, $(B)$ the design surface of one building bay façade module, $(C)$, the design behaviors of the agent based fenestration, and $(D)$ the analysis surface with the virtual sensor points of our experimental case study.

Three different types of a non-structural building component --façade panels that comprise our nonstandard fenestration scheme- are initiated by user (designer) defined geometric properties, such as length, width (extrusion), thickness, and curvature (Figure 2C). Starting points and direction for seeding the component pattern growth are defined by the designer (Figure 7E). The building façade bay is the design domain boundary, while the number and size of the openings - areas for unobstructed views- are demarcated as areas where the components cannot be placed (Figure 6E). A 
construction sequence is then simulated by placing one component after the other sequentially that incorporates the agency of fabrication and erection constraints; ensuring that they do not self-intersect as well as ensure that the whole façade remains an interconnected structure covering the whole design surface (Figure 2B). Each component type is based on a probability factor (explained in Section 3.6). The interior of the office is analyzed through 2623 virtual sensor points (Figure 2D), which measure light intensity within the space at height levels that the designer can specify (i.e., floor level, table top level). These virtual sensor points exist within the simulation environment of our analytical feedback loop. The generated designs are simulated and analyzed environmentally and their performance is then coupled with user preferences [25].

\subsection{The MAS Agent Design, Structure and Hierarchy}

Four different classes of agents are modeled in our system, with actions, properties, states and goals. Our agent classes include: (1) a generative design agent that encodes the design intention and geometric properties of the building component and is responsible for generating façade panel fenestration patterns that regulate the amount of light that enters the office space (described above); (2) specialist agents with a number of different sub-classes (based on the types of analysis) for analyzing and evaluating the generated designs' performance; and (3) a simulation agent that is responsible for simulating user preference and updating the generated designs accordingly. Lastly, (4) a coordination agent ensures each agent is aware of other agents' states and is responsible for the communication and coordination of the different classes. For the agents in the system, it is important to note that the behavioral rules vary in complexity and levels of information taken into account during the decision making process. The interaction patterns between agents and different design parameters or analyses are identified and transformed into negotiation and trade-off processes among them. Hierarchy among the agents is established and applied by a coordinating agent that communicates and controls the rest of the agents. Figure 3 illustrates the basic structure of an agent and the established hierarchies among the system's agent classes. Each of the aforementioned properties of our agent are then expressed as layers within the internal structure of an agent. Thus the established typical agent structure in this work includes: a) an interface layer through which the agent communicates with their environment; b) a definition layer which describes the set of states and goals of each agent; c) an organization layer which reasons the type of actions to be taken by the agent at a given time based on analytical data; d) a coordination layer which keeps track of past and current decisions; and finally e) a communication layer which establishes that the agents are communicating properly among themselves.

TYPICAL AGENT STRUCTURE

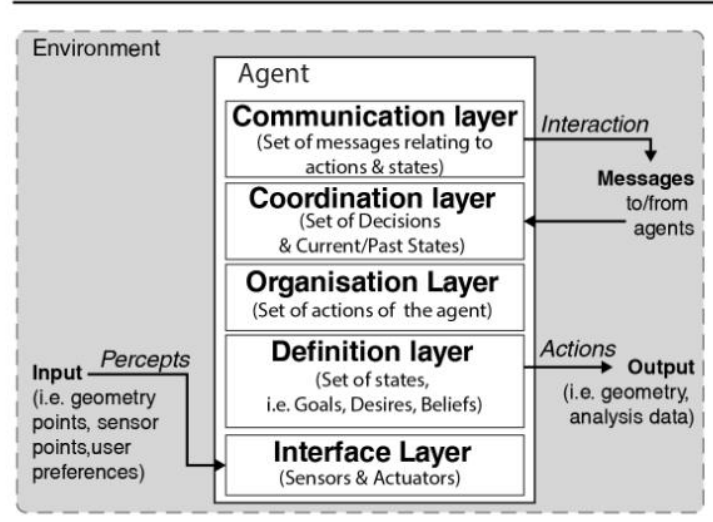

\section{AGENT HIERARCHY WITHIN MAS}

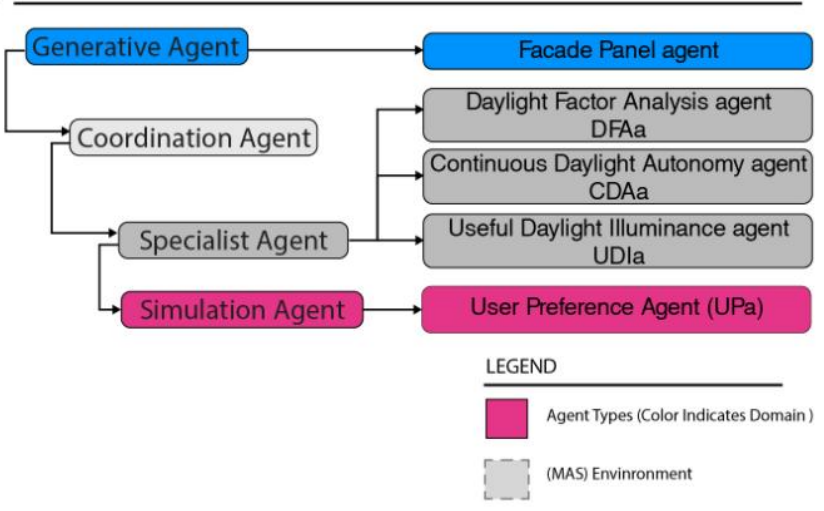

Figure 3: Diagram illustrating the typical agents' internal structure, agent types and agent hierarchy within the MAS for design.

Our MAS approach is developed in two stages; one the generative aspect of design, where agents act autonomously; and two, the optimization of generated outcomes, where agents act collaboratively and 
negotiate to find optimal solutions. Our system explores these complexly coupled relationships between and internal to the generative and analytical design processes. First, a set of generative agents and behaviors are modeled and instantiated, based on a given design site's location and orientation, a building façade bounding context, and designer defined parameters that append to building components (length, width, thickness, type). Initially, the systems' agents act autonomously and develop design alternatives, which satisfy local rules and constraints from the geometric domain, avoiding specific areas that are reserved for window openings and views, and collision checking for constructability. During a second loop, the designs are analyzed by a set of specialist and user preference agents, which communicate their data back to the generative agents in order to adjust parameters to regenerate design alternatives based on specific user preferences and performance goals.

\subsection{Description of Agent Functionalities}

\section{Generative Agent}

The generative agent type is the algorithmic design block of the MAS (Figure 4); with properties and geometric variables custom developed by the designer based on a given design brief. Its environment is the geometric domain, a NURBS surface, and its behavior includes generating geometries (i.e., the façade panels) on the input geometric domain to provide design alternatives that conform to the given specifications and satisfy the constraints in a near optimal solution. Near optimal, are considered the solutions that provide light closer to the user preference targets and that increase the availability of natural light (i.e., reducing energy demand from electric light). The agents are goal-oriented and their utility function is based on an initial design scheme (i.e., fill a surface with panels), in which the parameter ranges can be specified by the designer and/or building regulations. In this study, the generative agent is responsible for generating façade panel configurations in an automated fashion which satisfy the following criteria: stay within the given geometric domain, cover the domain by using a user defined number of panels and percentage of panel types, avoid specific areas that are reserved for openings, and provide an interconnected structure, ensuring constructability.

\section{Specialist Agents}

The specialist agent types are defined based on the types of analysis to be performed on the initial generated design (Figure 4). The environment of these agents is comprised of the generated designs and their task is to perform (environmental) analysis and communicate the results to other agents. The structure of the specialist agents is modular so that the analytical process can be performed by an (external) analysis software and can be extended to other design domains (e.g., structural analysis), which is work being conducted in a parallel set of experiments [20]. The agents work collaboratively and negotiate their utility functions locally in order to provide globally optimal solutions. Based on each performance metric the system for a particular iteration is defined through three types of specialist agencies: Daylight Factor Analysis agent (DFAa), Continuous Daylight Autonomy agent (CDAa) and Useful Daylight Illuminance agent (UDIa). DFAa analyses and communicates information relating to the daily and annual solar radiation for a given location and orientation. The DFAa evaluates the proposed designs and offers alternatives when direct solar radiation values within the space are over a certain limit and light distribution is too uneven. CDAa is responsible for configuring sub-areas within a space to have a certain level of Daylight Autonomy. The agent returns suggestions in the form of a .txt file for increasing the points that obtain a high percentage of Daylight Autonomy by adjusting the values of the key design parameters. The key design parameters are defined as having greatest impact on the specific analytical results. UDIa is responsible for increasing the area that obtains desirable UDI values. The agent returns suggestions (as a .txt file) on how to increase UDI in specific areas and levels (e.g., working level, eyesight level etc.) by adjusting the panels' design parameters (i.e., extrusion) locally.

\section{Simulation Agents}

The simulation agent type is defined by the user preference data collected (Figure 4). Their objective is to iteratively negotiate the adaptation of a design scheme and converge towards more optimal solutions that comply with the initial design requirements, while satisfying the user preferences. Currently the system relies on a fixed User Preference Agent (UPa) model. The UPa is tasked with 
profiling users and updating generated designs to better satisfy their preferences. The agent returns suggestions on how design parameters can be adjusted to meet daily usage patterns.

\section{Coordination Agent}

The coordination agent type is the communication controller of the system, and thus is responsible for setting up the hierarchy among the specialist agents, and for managing the communication among generative and specialist agents, ensuring that all agents are aware of other agents' states (Figure 4). The objective of this agent is to make sure every other agent is able to perform and has completed its task, as well as update the state of each agent at every step and deliver messages/reports on how to update their individual properties. This agent has an overview of the states of all other agents and therefore handles the tradeoffs among different agent types/ populations based on the data it collects and a heuristic function, which is defined below.

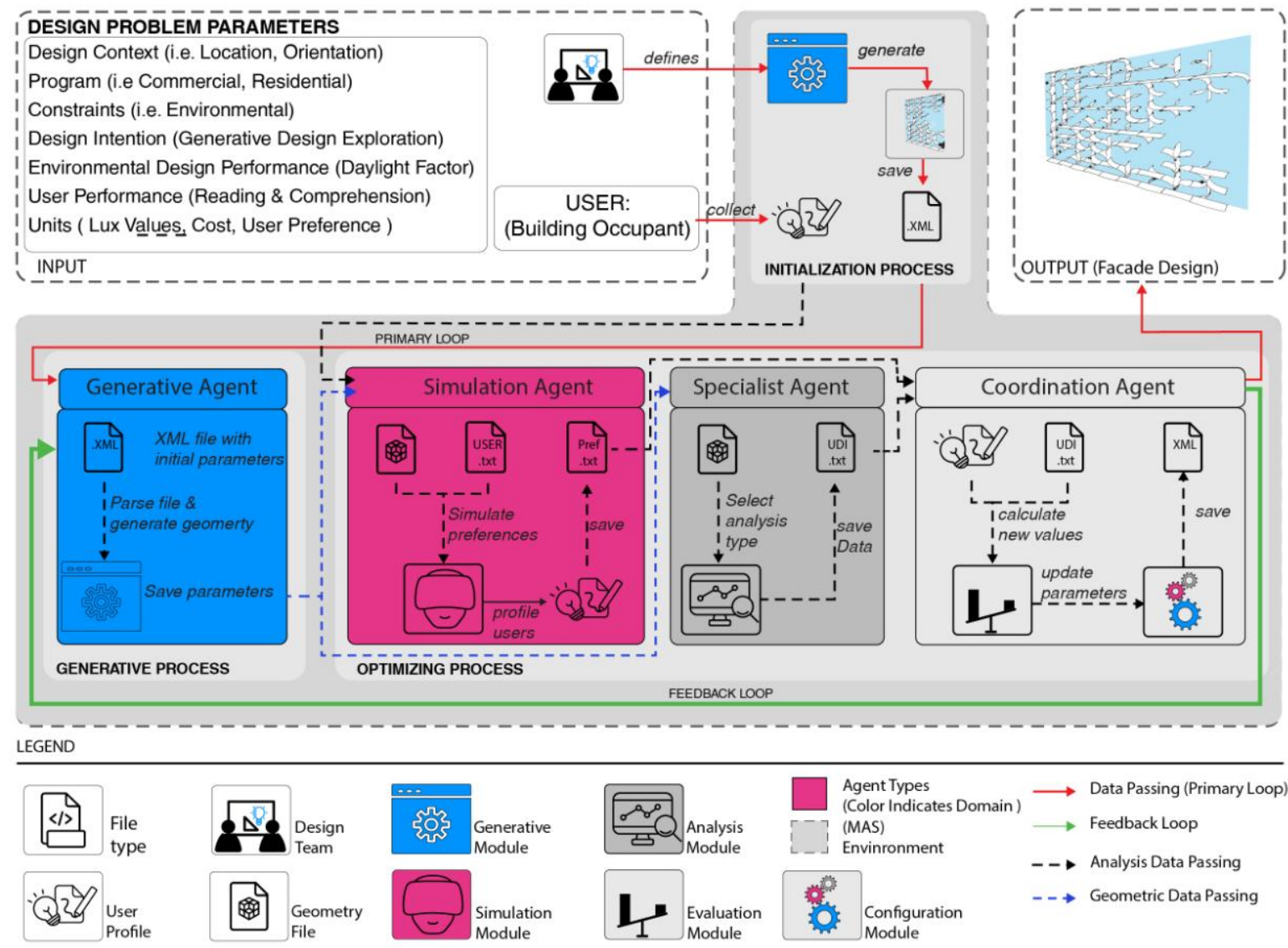

Figure 4: Diagram illustrating the MAS system with all domains (generative, simulation, analysis, and evaluation), sub-systems (agents), their relationships, feedback loops, and data passing amongst the agents. Within each domain the agent (sub-system) the performed processes are described graphically as geometry generation, environmental simulation and analysis, generative processes, optimization, and coordination.

\subsection{MAS for Design, Platform Architecture}

The prototype of our system is built on top of a number of open source software platforms and tools, as well as commercial software that offer open API's (Application Programming Interfaces). Our core bespoke agent types/classes of the system are developed in Java on the Eclipse Platform, which serves as the common interface for diverse IDE (Integrated Development Environment) based products to facilitate our integrations [57]. The custom programmed MAS utilize libraries and classes from Processing, a Java-based programming language with its own IDE [54]. For the geometric adaptation and transformation of the building components, the IGEO library is implemented, which has been developed to offer automatic data management of NURBS based geometry as agents, as well as 
method chaining for coding efficiency [68]. A custom java applet and a Graphical User Interface (GUI) are created to generate geometric configurations, which are then imported into a 3D NURBS design environment for further analysis (McNeel Rhinoceros). The designs are analyzed and evaluated using Grasshopper, a visual scripting editor within Rhinoceros and specifically, two Python based environmental simulation plugins, Honeybee and Ladybug. Automation functionalities are added via custom programming in Python in order to obtain and simulate environmental analysis from specialized stand-alone software (OpenStudio, EnergyPlus, Radiance, and Daysim). The obtained data is saved as .txt files, which are used to model and form the parameter bounds of an environmental set of agents. The Python language is used to handle the calls of the different platforms, while a 'federated' system architecture is used to relate multiple software environments. Extensible Markup Language (XML) is deployed to control and manage the agents properties and states, because it provides a flexible and adaptable information identification method that allows for designing a customized markup language for almost any type of document [62]. We have built the system with extensibility in mind, and with the goal of adding other domain constraints and objectives. Figure 5 illustrates all the system architecture and description of the platforms used and the corresponding relationships within our system.
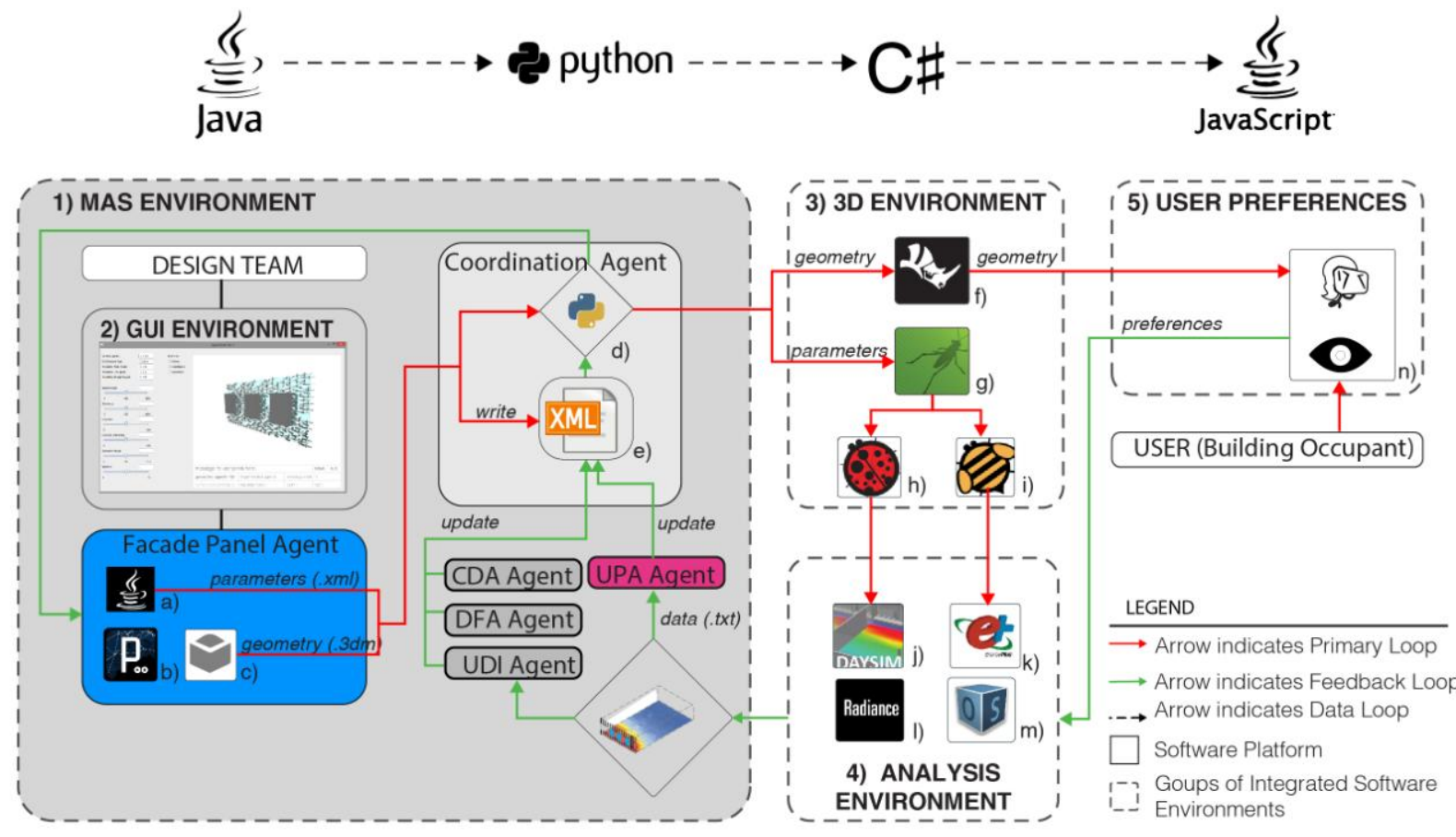

a) Oracle Java 7.0 Environment b) Processing Integrated Development Environment (IDE) c) IGeo NURBS library d) Python (IronPython, RhinoPython) e) Extensible Markup Language, f) Rhinoceros 3d: Nurbs modeling software g) Grasshopper (GHX): Visual script editor plugin for Rhinoceros , h) Ladybug: Enviromental Analysis plugin for GHX i) Honeybee: Enviromental Analysis plugin for GHX, j) Daysim: Radiance-Based Energy Analysis Software (Stand-alone) k) Energy Plus: Building Simulation Software (Stand-alone), I) Radiance: Raytracing Software System (Stand-alone) m) Open Studio: Building Energy Modeling Software (Stand-alone), n) Occulus Rift: Virtual Reality Head Display

Figure 5: MAS for Design platform architecture: MAS environments, inputs, data transfers among software platforms, as well actions and relationships among agents.

\subsection{Coordination, Evaluation and Optimization}

Communication and negotiation mechanisms among the agents are established in order to update the behavior of the generative agent(s) and improve their geometric results. Text file messages update values and/or actions to negotiate across different agents. At each iteration, the agents check the state of other agents, report their state, calculate their utility and predict future actions based on the utility. The effect of their actions and behaviors on other agents is dependent on the hierarchy established among the different agents (i.e., generative agent is higher in the hierarchy than the specialist agent) or the degree of importance of each agent's related behavior (i.e., user preference and environmental analysis) (Figure 3). The target of the negotiation for each agent is to satisfy its own goal while minimizing the negative side effects on the other agents. In other words, the specialist agents try to 
improve the environmental performance of the space by increasing the amount of natural light entering the space (i.e., increase the Daylight Autonomy Factor - Figure 6) while the UPa will try to maintain the light levels closer to the user(s)' most preferred setting. In order to optimize the performance of the space in terms of energy efficiency, we implement a hill-climbing algorithm [48], which is performed by the DFAa. At each step, the DFAa is responsible for one action from the following: move the center (i.e. position) of the opening in $\mathrm{x}$ or $\mathrm{y}$ direction, and/or increase/decrease the size of the window. The center of the opening can move within the domain of a given surface while the size of the window can increase/decrease up until a user (designer) defined limit. For this experiment the size of the solution space is a factorial of positions for $n=2$ openings times the grid points on the domain of the analysis surface, equaling 4950 iterations. The systems objective is to achieve a configuration of opening positions and sizes that offers higher levels of daylight across four date extremes, the solstices and equinoxes. The best performing results are selected by the CDAa based on the continuous daylight autonomy values and are passed as input to UPa. UPa uses the lux values at each virtual sensor point as an input while its objective function alters the design parameters (extrusion and angle) in order to minimize the deviation between the simulation results (lux values) of the best performing results (i.e. the Daylight Factor Analysis) and the simulation results (lux values) based on the user preferences. This is achieved by mapping the sensor points back to the façade panels as a feedback loop, and therefore, finding which panels have the most impact on the analysis surface. Each sensor point is mapped to multiple façade panels, which get a weighted value based on their impact to the sensor point. At each iteration, a parameter of the panel changes and a credit (i.e., +1 ) is attributed to each panel if the obtained value at the sensor point with highest impact is closer to the user preferred value. If the obtained value is further than the user preference a penalty is attributed (i.e., -10). Based on this credit or debit the agent can decide how to change a design parameter.

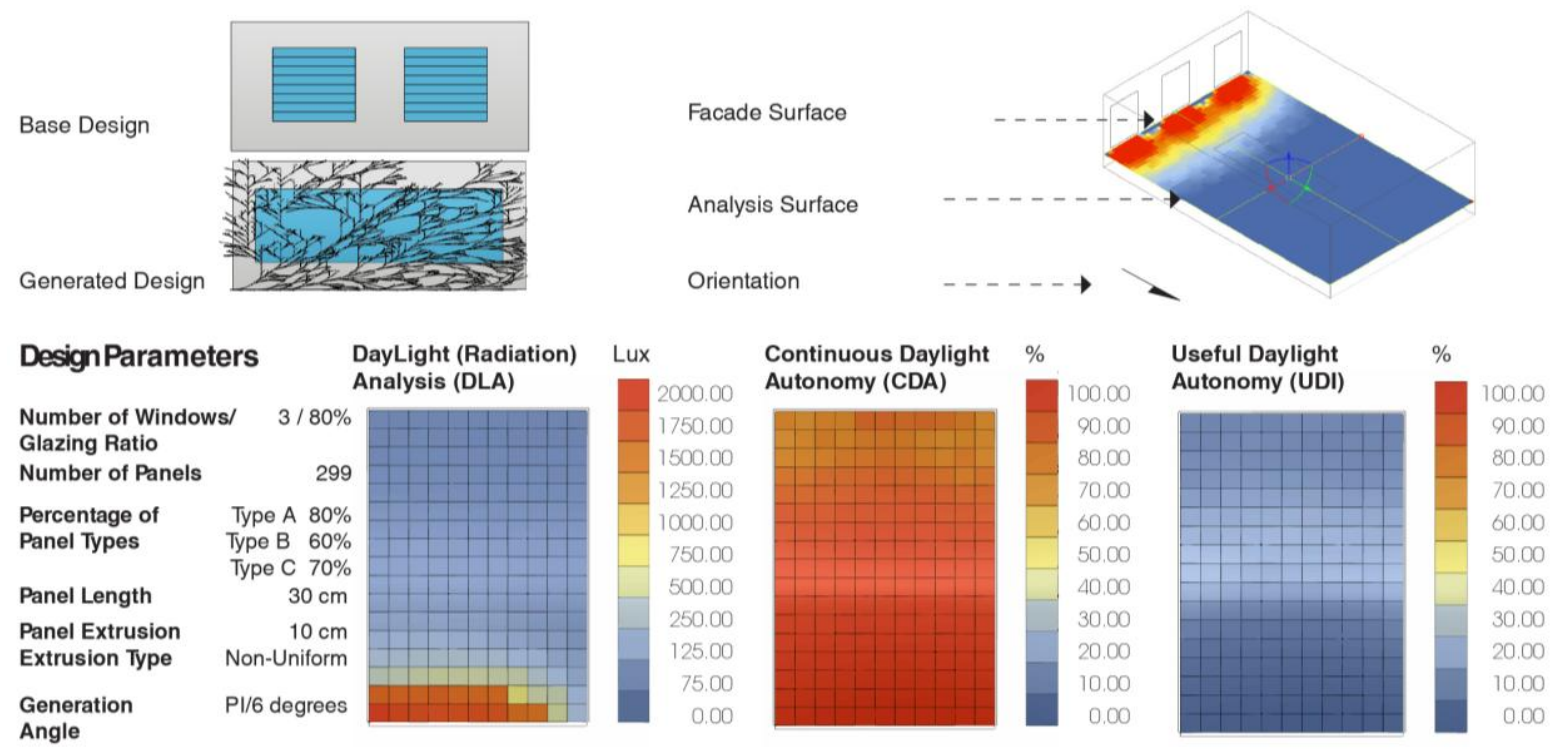

Figure 6: The benchmark study and normative fenestration example of a MAS generated façade (top left); the relationship between design surface and analysis surface (top right); main design parameters and types of environmental analysis (specialist agents) (below).

\subsection{MAS for Design, the Protocol and Workflow}

The design protocol and workflow has four phases. In the first phase, the generative agent iteratively grows $2 \mathrm{~d}$ lines on the panel surface trying to avoid areas that are reserved for specified window openings. In the second phase, the lines are transformed into $3 \mathrm{~d}$ surfaces via extrusion, finalizing the window panel fenestration pattern. In the third phase, the position and size of the openings are changed, triggering the regeneration of panel pattern configurations. At each generation design parameters that effect the environmental analyses the most, such as the depth of the panels are kept constant. In the last phase, the aforementioned design parameters are altered in order to optimize the environmental analysis to converge towards the user preferences. For the first phase, the parameters 
are: $L$, which defines the length of each line; $p 1, p 2, p 3$, which are the probabilities of each agent's behavior, connection angle between the lines, maximum number of agents as well as the number, size and position of the openings. For the second phase, the designer specifies $d$, the maximum extrusion length, and $\theta$, the maximum offset in the vertical direction. Hence, the lines are not only transformed into $3 \mathrm{~d}$ surfaces according to the length, but also are designed to be able to twist in order to better filter the light. In the third phase, the position and size of the openings change in order to find an optimal configuration for bringing more direct light into the space. This provides for a greater variety in facade design but also requires greater computational analyses to evaluate and optimize the nonstandard; hence why we focus on the highly idiosyncratic as an initial use case. In the fourth phase, a new type of specialist agent is created for each of the analyses: the DFAa, CDAa and UDIa. The designer specifies a utility " $u$ " for each of the performed analyses and the user preference profiles, which defines the most important analysis, adding a weighting factor to the agent's behaviors. The weighting factor is calculated as a percentage over all performed analysis types depending on the performance target that the designer sets. All of these aspects affect the amount (measured in lux) and type of sun light (direct or indirect) that enters the room, changing the illumination inside the space.
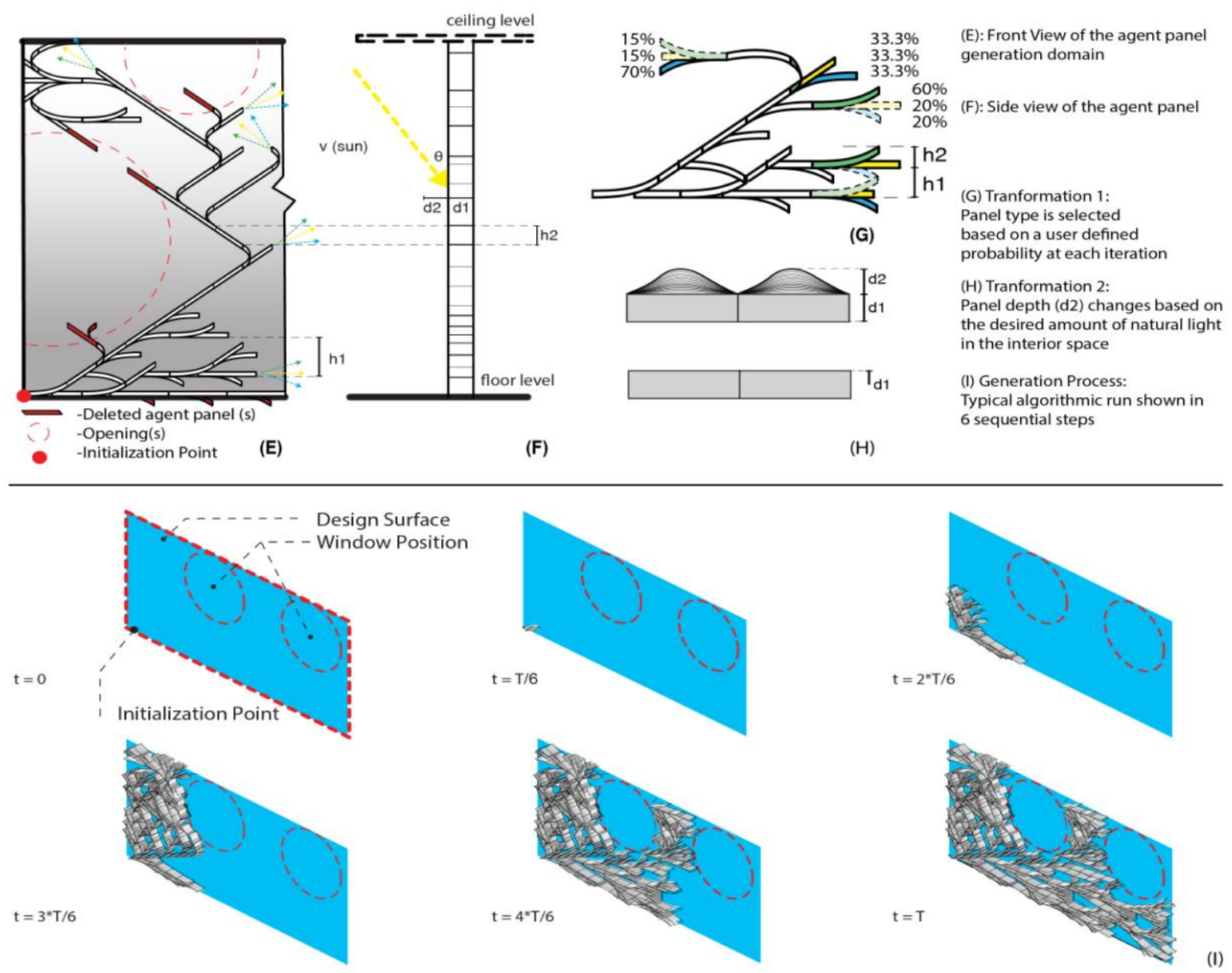

Figure 7: Rules of the generative façade panel agent(s) (E-H) and a typical generation process (I) shown in six sequential steps (from $t=0$, to $t=T$ ) including representative window openings.

As shown in Figure 7(I) in the first phase of the algorithm, the agent starts at a user defined initial point on the panel, and performs a series of iterations. At each iteration, the agent grows one line from its current position, and moves to the end of that new line (Figure $7(\mathrm{G})$ ). The agent can grow three different types of lines, according to three different behaviors: straight, left-curved, or right-curved with based on an angle $(\theta)$, as shown in Figure $7(\mathrm{G})$. In the beginning of each iteration, the agent picks its next behavior according to the (user) specified probabilities $p 1, p 2$ and $p 3$. However, the agent must also obey four constraints: (1) the new line must not intersect a previously constructed line, (2) the agent must not leave the boundaries of the given surface, (3) the agent must avoid specific 
areas reserved for openings, and (4) the amount of generated agents cannot exceed a maximum number specified by the designer. If the probabilistically chosen behavior violates these constraints, a new behavior of left, right, or straight is selected until valid. More specifically, the agent checks the history of all previous behaviors, as well as, the life value of each agent and changes to the behavior that has the ratio furthest away from the desired one according to the given probabilities $\mathrm{p} 1, \mathrm{p} 2$ and $\mathrm{p} 3$, which naturally induce a ratio. The above probabilities are being scaled based on the life state of the agent, which is influenced by whether the agent was created in a valid area (i.e., if agent is created in non-valid area life state decreases significantly).

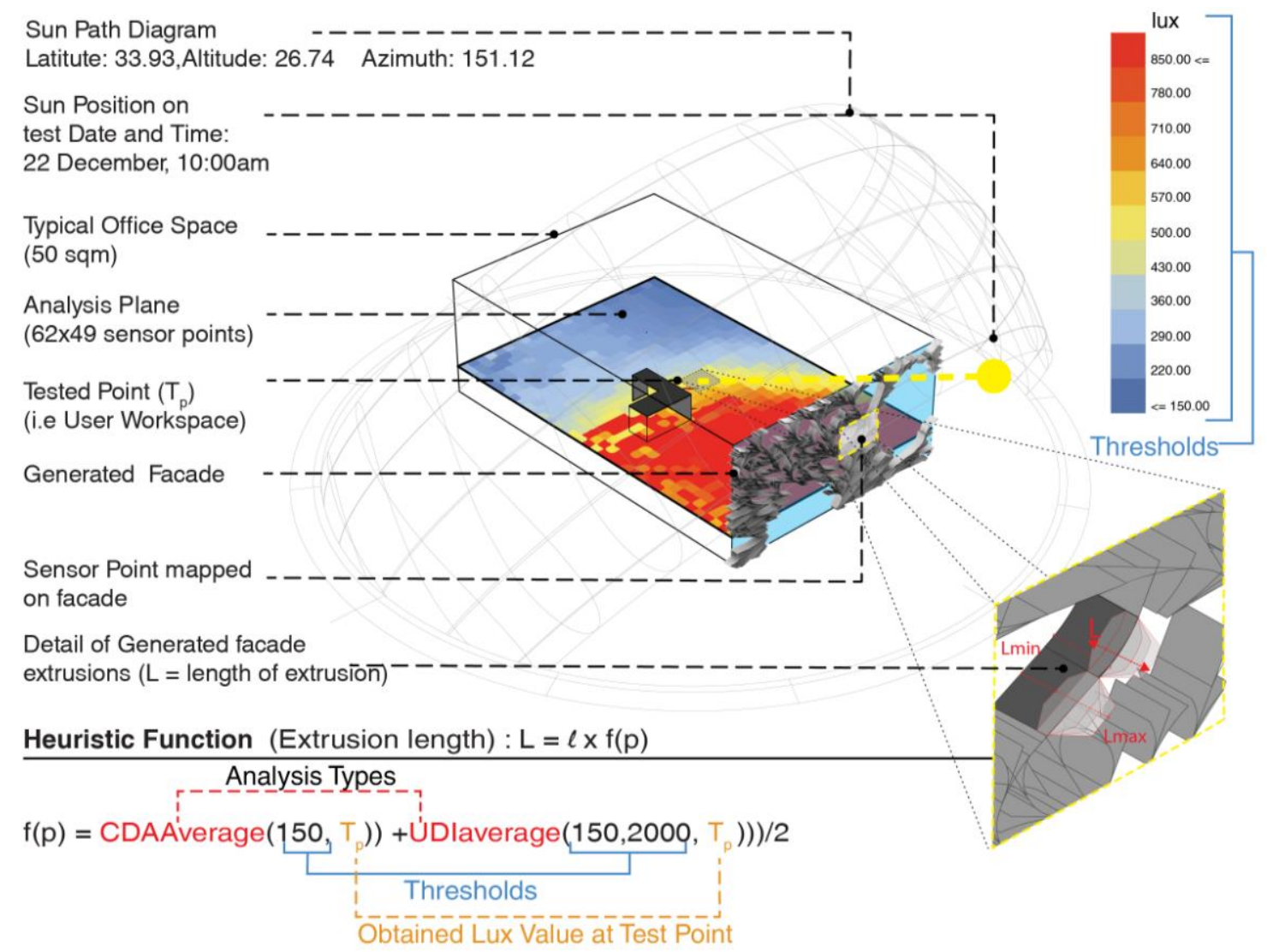

Figure 8: Experimental Design set up that describes, the environment, design parameters and the heuristic function that couples the extrusion of each façade panel with the analysis plane based on the degree (percentage) that each panel affects each specific virtual sensor point on the analysis plane (inside the office space i.e. floor or desk height).

This phase is terminated either after a pre-specified number of iterations is reached or if the maximum number of agents has been created. In the second phase, shown in Figure $7(\mathrm{E}-\mathrm{H})$, the lines are extruded into $3 \mathrm{~d}$ geometries. For each line, a length and angulation are chosen according to the following equations: $\mathrm{d}^{\prime}=\mathrm{d}^{*} \mathrm{w} ; \theta^{\prime}=\theta^{*} \mathrm{w}$, where $0 \leq w w \leq 1$ is a weight given by the current solar radiation entering the panel in the position of the line. Hence, each line will have a different d' and $\theta$ ' bounded by the preference of the user. The designer can specify two different types of extrusion: uniform or non-uniform (Figure $7(\mathrm{H})$ ). The non-uniform case differs from the previous description in that the user can also specify a "control point", which affects the "degree" of the curves, generating the surface as shown in the Figure 7(F). Finally, these parameters define the aperture $a$ ' between surfaces (Figure 7(E)), which in turn influences the amount and type of light that enters the space. In the third phase, the designer assigns, (1) specific set of days throughout the year (i.e., summer, winter solstice), (2) minimum and maximum desired luminance obtained for the majority of the sensor points inside the office, (3) specific time-period throughout the day (i.e., 9:00 am-5:00pm) as properties for the specialist agents (DFAa, CDAa, UDIa) where the goal is to find the optimal combination of generated façade panels, and (4) positioning of $n$ openings in order to provide luminance within the 
desired thresholds. The specialist agents (DFAa, CDAa, UDIa) for each run collectively, compare across and search the whole solution space for possible positioning ( $\mathrm{x}, \mathrm{y}$ as circle center points) of a number of openings ( $\mathrm{n}=2$ in this data set) on a given surface. The goal is to generate façade panel configurations that provide natural light availability above a designer defined level (i.e., CDA>150 lux) and closer to a user defined level (i.e. $150<\mathrm{UDI}<1200$ ). A run is then automated for an annual simulation, which then calculates the amount of natural light availability on the specific dates as well as the average values throughout the year. The optimal and sub-optimal solutions of each run are selected and passed as inputs for the next phase along with the corresponding analyses to the CDAa and UDIa whose goals are to alter specific design parameters (i.e., extrusion) in order to improve the environmental performance of the design. A heuristic function is defined for changing the design parameter based on the analysis values as such:

$$
\begin{gathered}
\operatorname{Extrusion}(L)=l \times f(p) \\
f(p)=\frac{[\text { CDAAverage }(\text { Threshold } 1, T) \pm \text { UDIaverage }(\text { Thres } 1, \text { Thres } 2, T)] \pm \text { credit }}{2}
\end{gathered}
$$

Finally, the UPa takes as input the best ranking designs in terms of the CDAa analysis and combines them with user preference data, and attributes a credit (described in Section 3.5) to each agent, which is proportional to how much the analysis on each virtual sensor point deviates from the user preferences (Figure 8). At this stage, the user preferences reflect the preferred lux value of a user. Based on this utility factor, all the above parameters are recalibrated and agents iteratively negotiate in order to meet the performance values with higher utility while ensuring the minimum amount of conflicts with the other specialist agents.

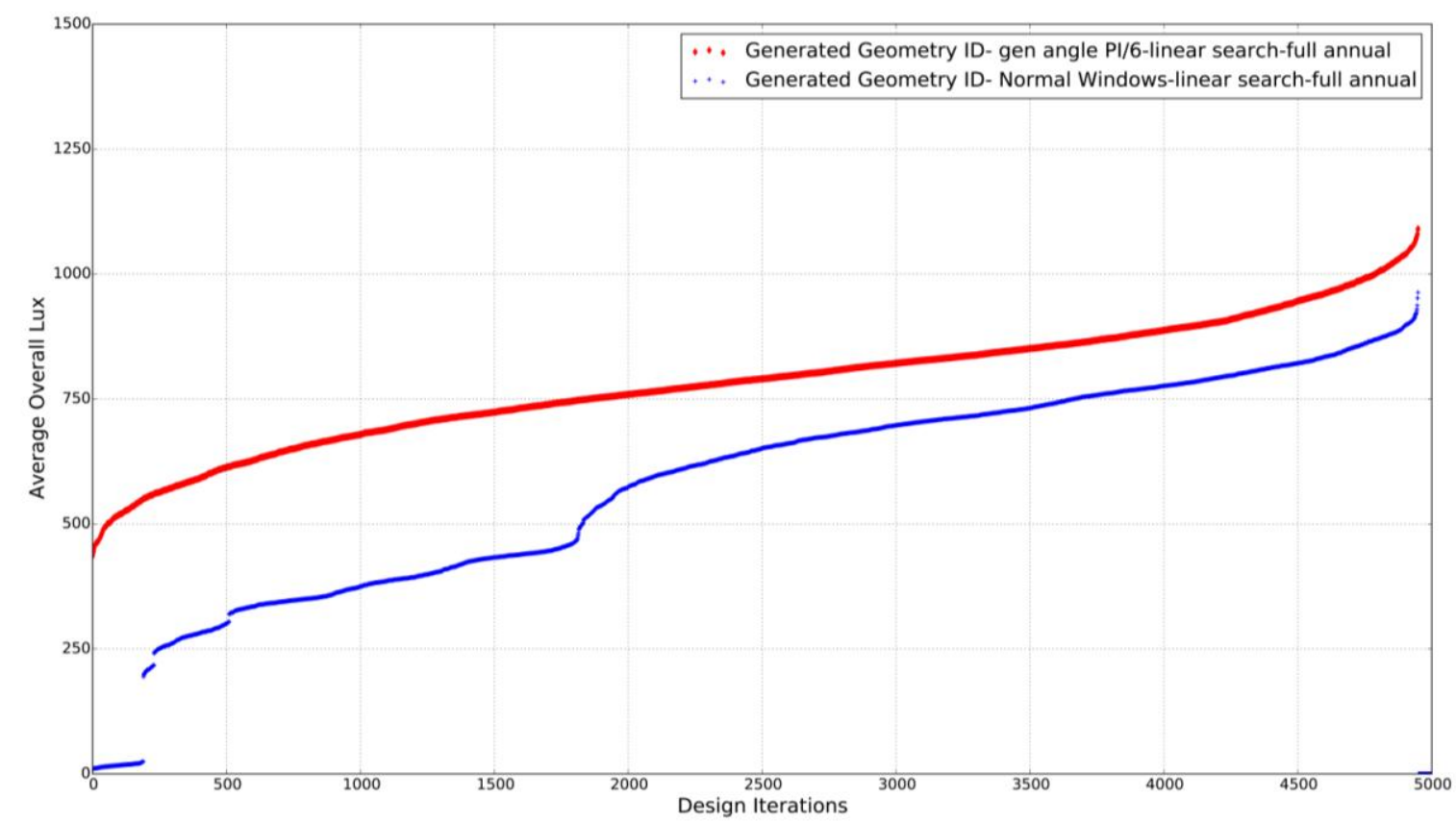

Figure 9: Comparative plot of the Average Daylight Factor Analysis (DFA) for the 4950 unique design iterations (red) and the benchmark study with normal windows (blue) for 4 key dates $(22 / 3,22 / 6,22 / 9,22 / 12)$.

\section{RESULTS AND ANALYSIS}

For this experimental design case the generation of alternative façade panel configurations is based on all the possible positions for $n=2$ openings on a bay of the south façade of an office building in Los Angeles. The results of the experiment conducted are based on the generation, analysis and evaluation of unique façade panel configurations. As a first step in testing our system, we searched the whole 
solution space of all the possible locations of openings on the façade with the aim to' (1) test the generative aspect and robustness of our system at present shown in Figure 11, and (2) deduct a mathematical relationship (our heuristic function) between the position of the windows, the pattern of the façade and the natural light availability, at present shown in Figure 9. Thus, we divided our design surface into a 10x10 grid (100 points) and performed a linear search for all possible locations of openings $(n=2)$ with a fixed size. This resulted in the generation of 4950 unique façade panel configurations, with varying panel type percentages but with a single generative angle (PI/6) and uniform length $(0.05 \mathrm{~m})$. In order to test the generative aspect of this bottom up approach and for ensuring relevance to contemporary architectural design and engineering challenges, we purposefully developed a simple branching fenestration system that can aggregate into complex façade configurations (as can be seen in Figures 7, 8, and 10). The generated design outcomes (façade panel patterns) were analyzed annually over a specific time-period (9:00 a.m. - 6:00 p.m.) and are compared against the baseline case study with normative window openings (Figure 9). From the plot in Figure 9 comparing the base case to the experimental case with $n=2$ openings, we observe that the system generated façade configurations (red line), perform better than the baseline case study (blue line) for all window positions, as they obtain on average 100-150 lux of more direct sunlight annually. The system was also able to produce unique designs solution for each given combination of position and size of the $n=2$ openings. In addition, for specific window positions the performance of the baseline study is significantly low and non-continuous, whereas for the MAS generated façade configurations there is a consistent relationship between the window positions and the obtained lux values as seen in the continuously optimizing curve. Through the incorporation of the environmental analysis into the agents and the introduction of ABMS negotiations among their behavior and the user preferences, we anticipated the generation of unexpected design solutions based on the adjustment of the desired analysis. Specifically, we measured and compared the following analyses: (1) average annual daylight radiation (DFA) in Lux; (2) central daylight autonomy (CDA) as a percentage of area with light values above 150 lux; and (3) useful daylight illuminance (UDI) as percentage of area with light values between 150 and 1200 lux which in our case represent the preferred lighting condition of the selected group of users (Figure 10). It is important to note Figure 10 represents a cumulative improvement of the simulated designs, as CDA and UDI results are performance metrics based on the DFA, which is shown to be improved in Figure 9 when compared to the base case. Figure 10 illustrates the plots that correspond to each of the above analysis, as well as a subset of best (images on the right) and worst (images on the left) performing generated geometries in relation to each metric. The results are sorted according to the improvement of the analysis for each design iteration. The system was able to generate façade configurations that obtain above 450 lux on average and that obtain a CDA within the range of $85-93 \%$ for the tested time period, across the whole surface area of the office. The results showed that $1 \%$ of the generated geometries achieved a UDI above $40 \%$ across all dates, without being optimized by the UPa, which is in part a focus of the next set of experiments. The curvature of the graph in all four plots implies a mathematical relation between the window openings, the paneling system and the obtained analysis, which we continue to investigate. Figure 11 illustrates a subset of optimal solutions on the pareto frontier in relation to both CDA and UDI. The illustrated pareto frontier is a subset of the design space in which UDI and CDA are the focus of the graph, which is explainable as lower versus higher positioned windows have the greatest effect on this metric of performance, as one would expect at the fourth story. From the experimental data, we conclude our system achieves the following: (1) ability to generate unique outcomes based on different context conditions (unobstructed view locations); (2) it provides design outcomes that perform within a given bounded light range (measured in lux); (3) it provides $45 \%$ of design outcomes that operate without the use of artificial light for a specific time period given a minimum light intensity; (4) and it produces $6 \%$ of design solutions that operate without the use of artificial light (in order to maintain an illuminance larger than 150 lux) while at the same time complying with the preferred values defined. 


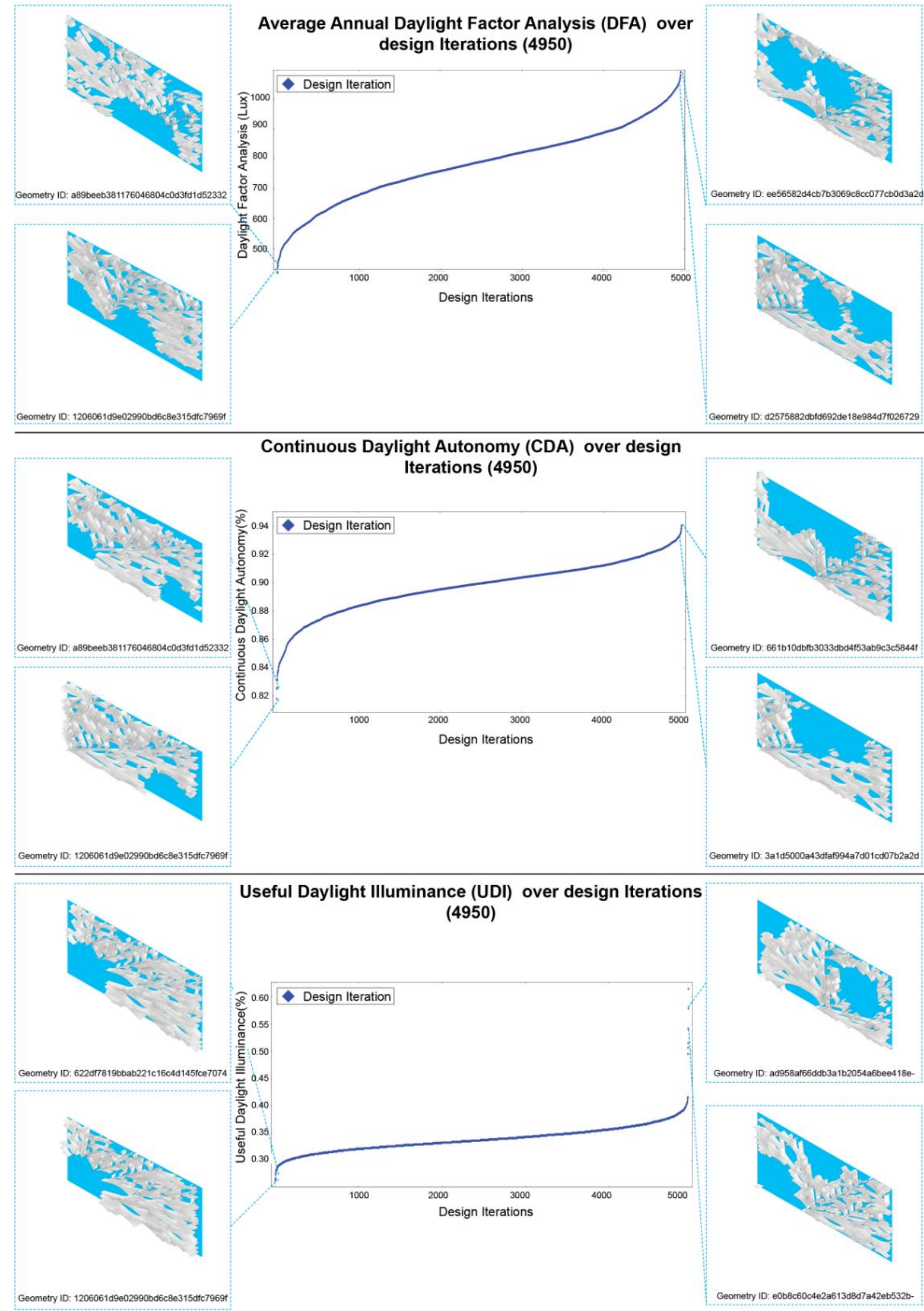

Figure 10: Data plots illustrating the 4950 unique design iterations in relation to Average Annual Daylight Factor Analysis (DFA, top), Continuous Daylight Autonomy (CDA, middle), Useful Daylight Illuminance (UDI, bottom), and the 2 best (right) and 2 worst (left) ranking design solution solutions in each case. 


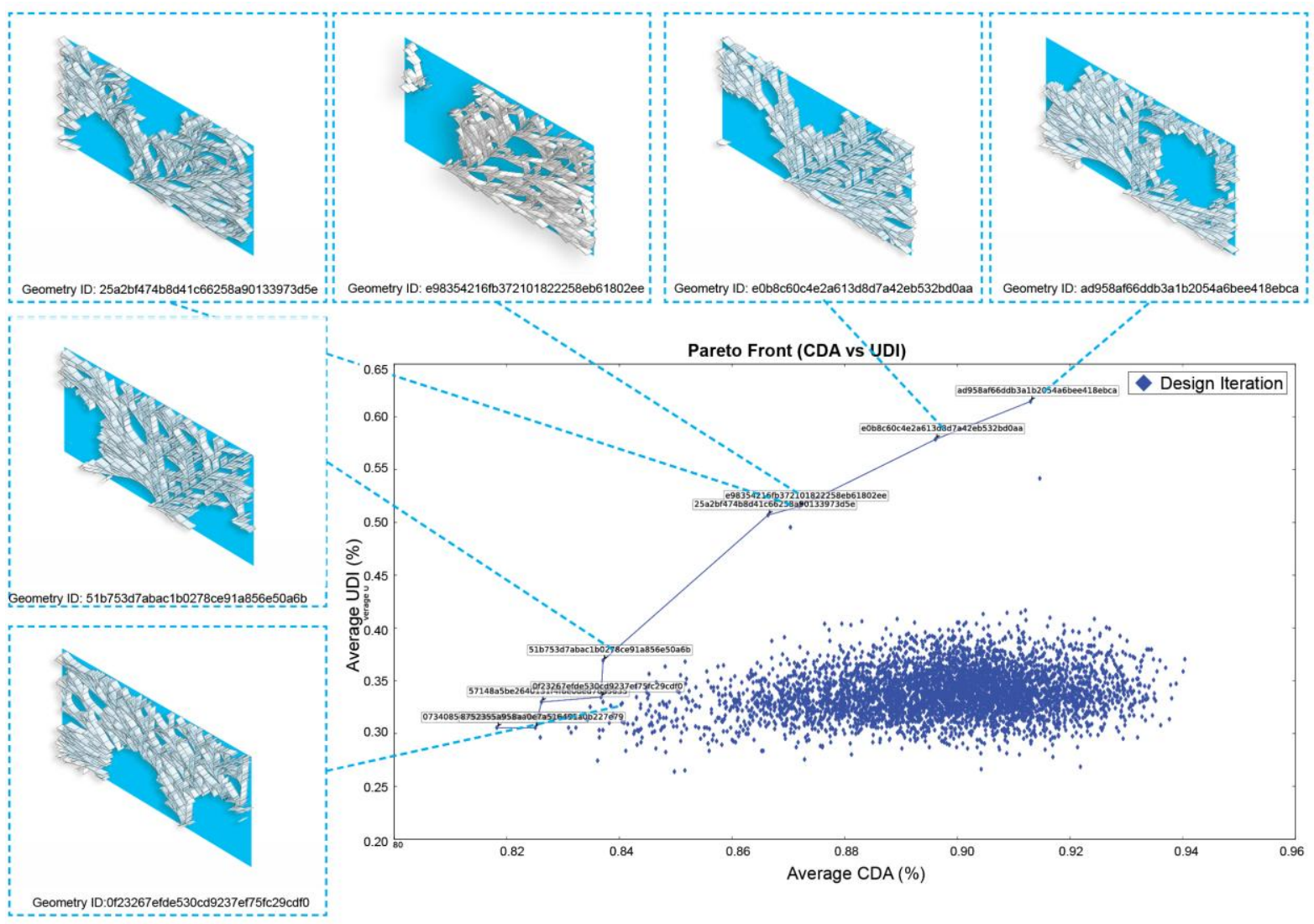

Figure 11: The pareto front of the combination of two types of analysis (CDA and UDI) and a subset of the geometries that perform best through increasing the amount of available natural light $(>150$ lux) while meeting the user's preferences.

\section{LIMITATIONS AND FUTURE WORK}

The limitations of the experiment include the fact that we applied our system on a building, which is located in an area with little climatic variance and no extreme conditions. We believe this to be less a limitation but rather a reason to extend our experimental design and one of our future tasks. Although the system has been developed in way that can be easily scaled up, we only tested our system on a sub region of the building's façade with a given orientation (south) and thus we intend to test how the system behaves on a larger scale and for varying orientations, this is already in progress. At this stage of the research, heat gain is not accounted for in the office space, which does relate to increased availability of natural light. Additionally, the system was tested for only one specific user preference group and did not account for multi-occupancy with varying light user preferences; this is a planned future task. Lastly, the simulated data of the users reflect their light preference for a specific time of the year which we extrapolated as their preference for the whole year, this will be made dynamic in future experiments.

Apart from the above stated limitations, the development of our custom MAS for design system posed a set of technical challenges, such as how to define the agents' types/classes based on design requirements for use on other design typologies, such as for our parallel research on structural shells or for other designer driven geometries, standard and idiosyncratic alike. In addition, another challenge was how to develop utility functions for the agents in order to effectively communicate and negotiate between the generative and the optimization processes. In other words, how the analytical results could be properly formulated into agents' design actions and how to establish a smooth communication between the different platforms that have been used to collect the analytical data. So far, we have implemented environmental analyses and simulations for optimizing the generative design process, however, the general MAS for design method anticipates further extensions into structural and robotic fabrication arenas with direct feedback from those systems. Lastly, although we 
were able to analyze the generated designs of the solution space in terms of environmental performance, our interest in analyzing the diversity of the generated outcomes quantitatively is one of our critical next steps in order to measure how different the generated optimal designs were amongst themselves. To reiterate, the research targets architectural design relevance through two primary vectors; (1) that of aiding in the automating of constructible yet highly intricate designs; and (2) that of demonstrating that these intricate and geometrically unique designs with intrinsic complexity are inherently improving the performance.

As part of our future work, the research plan includes running the system on different locations, buildings and façade configuration to continue test the systems performance in different design scenarios. The research intention includes testing how the system performs; when multiple user preferences are combined within the same office space; and when applied to a whole building façade of both Euclidean and non-Euclidean geometries. Furthermore in terms of system development and calibration, we plan to include robotic fabrication simulations as additional factors for conditioning the generative process. Structural analysis is another optimizing process that is being integrated into the MAS for design to operate in conjunction with the environmental analysis. Lastly, we aim to further develop the GUI in order to assist the human designer interaction with the system and for validating its impact on design collaboration and practice.

\section{CONCLUSIONS}

This paper presents the application of a MAS for design approach that supports the integration of environmental performance data and user-related information in order to inform the design decision making of a generative design process, leading to better performing design solutions. The aim of this work is two-fold: (1) to explore how designers could implement non deterministic processes where the precise definition of local rules can be combined with analytical tools in order to lead to globally optimized geometries that also embody user preferences, and (2) to develop an integrated design methodology based on MAS that provides designers with not only geometric feedback and diversity but with multiple performance feedback and thus assist them in generating and selecting among well performing design solutions. The presented experimental case illustrates how a MAS for design can be developed and tailored to specific design problems in order to output design alternatives in an automated fashion and therefore assist architects and their collaborators in generating higher performing design solutions (i.e. higher lux values obtained on the analysis surface). The geometric configurations of the design solutions may vary but the coupling of the generative with the analytical and optimizing processes ensures the satisfaction of prescribed standards (i.e. minimum lux value for an office space) and specific user lighting preferences.

The system was able to generate façade panel configuration(s) exhibiting a high degree of complexity, which provided more natural light availability, specifically tailored to a specific group of participants, while decreasing the need for artificial light. We have and will continue to demonstrate that architects and designers can benefit by implementing an integrated bottom up design approach for the design of building components (post-Fordist and non-standard) that lead to optimal global configurations. By combining analytical methods with user data, properly formulating and passing such data automatically to a generative process, we provide designers with a larger pool of complex yet well performing design solutions that could not be modeled manually. The design case study and results showed improvements in terms of energy efficiency by generating solutions that increased the amount of available natural daylight (measured in Lux) and therefore infer a decreased energy consumption from artificial light. It also improves the design process by giving the designer the key role of describing the geometric DNA of a basic design unit (i.e., a building component i.e. façade panel) and their constraints and analysis objectives in which the system then automatically generates a series of global design solutions that are already incorporating the optimization of environmental objectives.

\section{Acknowledgements}

This material is based upon work supported by the National Science Foundation under Grant No.1231001. Any opinions, findings, and conclusions or recommendations expressed in this material 
are those of the author(s) and do not necessarily reflect the views of the National Science Foundation. We would like to give special thanks to all the participants and researchers that contributed to this project; specifically, to Arsalan Heydarian, Leandro Soriano Marcolino, Brian Herrera, Kevin Daley, Punit Das, Justin Yang and Francine Ngo for their contributions in preparing and running the experiments and in the illustrations of the results.

\section{References}

[1] Y.D. Abbasi, M. Short, A. Sinha, N. Sintov, C. Zhang, M. Tambe, Human Adversaries in Opportunistic Crime Security Games: Evaluating Competing Bounded Rationality Models, Proceedings of the Third Annual Conference on Advances in Cognitive Systems ACS, 2015, p. 2.

[2] H. Achten, J. Jessurun, An Agent Framework for Recognition of Graphic Units in Drawings, 20th eCAADe Conference Proceedings: Connecting the Real and the Virtual - design e-ducation, Warsaw,Poland, 2002, pp. 246-253.

[3] G.D. Ander, Daylighting performance and design, John Wiley \& Sons, 2003.

[4] C. Anumba, O. Ugwu, L. Newnham, A. Thorpe, A multi-agent system for distributed collaborative design, Logistics Information Management 14 (5/6) (2001) 355-367.

[5] C.J. Anumba, L. Newnham, 0.0. Ugwu, Z. Ren, Intelligent Agent Applications in Construction Engineering, Creative Systems in Structural and Construction Engineering, CRC Press, 2001.

[6] B. Aranda, C. Lasch, Flocking, Tooling, Vol. 27, Princeton Architectural Press, New York, 2006.

[7] E. Baharlou, A. Menges, Generative Agent-Based Design Computation, in: R.a.S. Stouffs, Sevil (eds.) (Ed.), eCAADe Conference-Computation and Performance Vol. Volume 2, eCAADe, Faculty of Architecture, Delft University of Technology, Delft, The Netherlands,, 2013, pp. 165-174.

[8] M. Bechthold, N. King, A.O. Kane, J. Niemasz, C. Reinhart, Integrated environmental design and robotic fabrication workflow for ceramic shading systems, in: I.A.f.A.a.R.i. Construction (Ed.), 28th International Association for Automation and Robotics in Construction (ISARC), Seoul, Korea, 2011, pp. 70-75.

[9] J. Beetz, J. Van Leeuwen, B. De Vries, Towards a Multi Agent System for the Support of Collaborative Design, Eindhoven University of Technology, Developments in Design \& Decision Support Systems in Architecture and Urban Planning, 2004.

[10] E. Bonabeau, M. Dorigo, G. Theraulaz, Swarm intelligence: from natural to artificial systems, Oxford university press, New York, USA, 1999.

[11] H.-J. Bullinger, W. Bauer, G. Wenzel, R. Blach, Towards user centred design (UCD) in architecture based on immersive virtual environments, Computers in Industry 61 (4) (2010) 372-379.

[12] P.M. Carranza, P. Coates, Swarm modelling: The use of Swarm Intelligence to generate architectural form 3rd Generative Art Conference, AleaDesign Publisher, Milan, Italy, 2000.

[13] C.-H. Chen, A prototype using Multi-Agent Based Simulation in Spatial Analysis and Planning, 14th Annual Conference of the Association of Computer Aided Architectural Design (CAADRIA), Douliu, Taiwan, 2009, pp. 513-521.

[14] J. Dijkstra, H.J. Timmermans, A. Jessurun, A multi-agent cellular automata system for visualising simulated pedestrian activity, Theory and Practical Issues on Cellular Automata, Springer, 2001, pp. 29-36. 
[15] P. Ednie-Brown, A. Andrasek, Continuum : A Self-Engineering Creature-Culture, Architectural design 76 (5) (2006) 18-25.

[16] V. Fabi, R.V. Andersen, S.P. Corgnati, B.W. Olesen, A methodology for modelling energy-related human behaviour: Application to window opening behaviour in residential buildings, Building Simulation 6 (4) (2013) 415-427.

[17] D.J. Gerber, The parametric affect: Computation, innovation and models for design exploration in contemporary architectural practice, Harvard University Graduate School of Design, Cambridge, 2009.

[18] D.J. Gerber, Parametric practices: Models for design exploration in architecture, Harvard University Graduate School of Design, 2007.

[19] D.J. Gerber, S.-H.E. Lin, Designing in complexity: Simulation, integration, and multidisciplinary design optimization for architecture, Simulation (2013) 1-24.

[20] D.J. Gerber, E. Pantazis, L.S. Marcolino, Design Agency, in: G. Celani, D.M. Sperling, J.M.S. Franco (Eds.), Computer-Aided Architectural Design Futures. The Next City-New Technologies and the Future of the Built Environment, Springer, 2015, pp. 213-235.

[21] J.S. Gero, Computational models of innovative and creative design processes, Technological Forecasting and Social Change 64 (2-3) (2000) 183-196.

[22] J.S. Gero, F.M.T. Brazier, Intelligent agents in design, Artificial Intelligence for Engineering Design, Analysis and Manufacturing (AIEDAM) 18 (2004) 113.

[23] J.S. Gero, V.A. Kazakov, An exploration-based evolutionary model of a generative design process, Microcomputers in Civil Engineering 11 (1996) 211-218.

[24] C.M. Herr, Generative Architectural Design and Complexity Theory, 5th International Conference of Generative Art, Vol. 16, Milan, Italy, 2002, pp. 1-13.

[25] A. Heydarian, J.P. Carneiro, D. Gerber, B. Becerik-Gerber, Immersive virtual environments, understanding the impact of design features and occupant choice upon lighting for building performance, Building and Environment 89 (2015) 217-228.

[26] A. Heydarian, J.P. Carneiro, D. Gerber, B. Becerik-Gerber, T. Hayes, W. Wood, Immersive virtual environments versus physical built environments: A benchmarking study for building design and user-built environment explorations, Automation in Construction 54 (0) (2015) 116-126.

[27] J.H. Holland, Adaptation in natural and artificial systems: An introductory analysis with applications to biology, control, and artificial intelligence, MIT Press, Cambridge, MA, 1992.

[28] J.H. Holland, Hidden order: How adaptation builds complexity, Basic Books, 1995.

[29] T. Ireland, Emergent space diagrams: The application of swarm intelligence to the problem of automatic plan generation, in: T. Tidafi, a.T. Dorta (Eds.), CAADFutures : Joining Languages, Cultures and Visions, Montreal,Canada, 2009, pp. 245-258.

[30] N.R. Jennings, J.M. Corera, I. Laresgoiti, Developing Industrial Multi-Agent Systems, ICMAS, 1995, pp. 423-430.

[31] Y. Jin, W. Li, Design concept generation: a hierarchical coevolutionary approach, Journal of Mechanical Design 129 (10) (2007) 1012-1022.

[32] J.S. Jordan, The exponential convergence of Bayesian learning in normal form games, Games and Economic Behavior 4 (2) (1992) 202-217.

[33] Y.E. Kalay, Performance-based design, Automation in Construction 8 (4) (1999) 395-409. 
[34] G. Kavulya, D.J. Gerber, B. Becerik-Gerber, 'Designing in' complex system interaction: Multi-agent based systems for early design decision making, ISARC 2011, \{Kavulya, 2011 \#94\}, Seoul, Korea, 2011, pp. 694-698.

[35] A. Kilian, Design exploration through bidirectional modeling of constraints, Department of Architecture, Massachusetts Institute of Technology, 2006.

[36] L. Klein, G. Kavulya, F. Jazizadeh, J. Kwak, B. Becerik-Gerber, M. Tambe, Towards optimization of building energy and occupant comfort using multi-agent simulation,, 28th International Symposium oo Automation Robotics and Construction (ISARC), 2011.

[37] L. Klein, J.-y. Kwak, G. Kavulya, F. Jazizadeh, B. Becerik-Gerber, P. Varakantham, M. Tambe, Coordinating occupant behavior for building energy and comfort management using multi-agent systems, Automation in Construction 22 (2012) 525-536.

[38] B. Kolarevic, Architecture in the digital age: design and manufacturing, Taylor \& Francis, 2004.

[39] B. Kolarevic, A.M. Malkawi, Performative architecture: Beyond instrumentality, Spon Press, New York, 2005.

[40] A.X.J. L. S. Marcolino, and M. Tambe. , Multi-agent Team Formation: Diversity Beats Strength?, In Proceedings of the 23rd International Joint Conference on Artificial Intelligence (IJCAI 2013), Beijing, China, 2013.

[41] N. Leach, Swarm Urbanism, Architectural design 79 (2009) 56-63.

[42] N. Leach, D. Turnbull, C. Williams, Digital tectonics, Wiley, University of Michigan, 2004.

[43] C.M. Macal, M.J. North, Agent-based modeling and simulation, Winter simulation conference, Winter Simulation Conference, 2009, pp. 86-98.

[44] M.L. Maher, M. Kim, Supporting Design Using Self-Organizing Design Knowledge, CAADRIA 2004 - Proceedings of the 9th International Conference on Computer Aided Architectural Design Research in Asia, Seoul,Korea, 2004.

[45] L.S. Marcolino, D. Gerber, M. Tambe, S. Veetil P, J. Musil, B. Kolev, S. Price, Agents vote for the environment: Designing energy-efficient architecture, AAAI Workshop on Computational Sustainability, Texas, 2015.

[46] U. Meissner, U. Rüppel, M. Theiss, Network-Based Fire Engineering Supported by Agents, Proceedings of the Xth International Conference on Computing in Civil and Building Engineering (ICCCBE-2004), Weimar, Germany, 2004.

[47] A. Menges, Computational morphogenesis, Proceedings for 3rd International ASCAAD Conference, Vol. 7, Alexandria,Egypt, 2007, pp. 725-744.

[48] M. Mitchell, J.H. Holland, When will a genetic algorithm outperform hill-climbing?, Proceedings of the 5th International Conference on Genetic Algorithms, Morgan Kaufmann Publishers Inc., San Francisco, CA, USA, 1993.

[49] W.J. Mitchell, The logic of architecture: Design, computation, and cognition, MIT press, Cambridge, MA, 1990.

[50] T. Mullen, M.P. Wellman, Some issues in the design of market-oriented agents, Intelligent Agents II Agent Theories, Architectures, and Languages, Springer, 1996, pp. 283-298.

[51] S. Parascho, M. Baur, J. Knippers, A. Menges, Design Tools for Integrative Planning, in: R. Stouffs, S. Sariyildiz (Eds.), Proceedings of the 31st eCAADe Conference, Vol. 2, Delft University of Technology, Delft, The Netherlands, 2013, pp. 237-246. 
[52] D. Phillips, Daylighting: natural light in architecture, Elsevier,Architectural Press, Burlington,MA, 2004.

[53] H. Pottmann, M. Eigensatz, A. Vaxman, J. Wallner, Architectural geometry, Computers \& Graphics 47 (2015) 145-164.

[54] C. Reas, B. Fry, Processing: A programming Handbook for Visuakl Designers and Artists, MIT Press, Boston, 2007.

[55] C.F. Reinhart, J. Mardaljevic, Z. Rogers, Dynamic daylight performance metrics for sustainable building design, Leukos 3 (1) (2006) 7-31.

[56] H. Rittel, M.M. Webber, 2.3 planning problems are wicked, Polity 4 (1973) 155169.

[57] J.D. Rivières, J. Wiegand, Eclipse: A platform for integrating development tools, IBM Systems Journal 43 (2) (2004) 371-383.

[58] F. Scheurer, Getting complexity organised Using self-organisation in architectural construction, Automation in Construction (16) (2007) 78 - 85.

[59] P. Schumacher, The autopoiesis of architecture, (2011).

[60] P. Schumacher, Parametric Order - Architectural Order via an Agent Based Parametric Semiology, in: T. Spyropoulos (Ed.), Adaptive Ecologies - Correlated Systems of Living by Theodore Spyropoulos, Vol. 1, AA Publications, London, 2013.

[61] T. Schwinn, A. Menges, D.0. Krieg, Behavioral Strategies: Synthesizing design computation and robotic fabrication of lightweight timber plate structures, ACADIA: Design Agency, Los Angeles, 2014.

[62] K. Shea, R. Aish, M. Gourtovaia, Towards integrated performance-driven generative design tools, Automation in Construction 14 (2) (2005) 253-264.

[63] D. Simeone, Y.E. Kalay, D. Schaumann, S.W. Hong, Modelling and Simulating Use Processes in Buildings, eCAADe 2013: Computation and Performance, Faculty of Architecture, Delft University of Technology; eCAADe (Education and research in Computer Aided Architectural Design in Europe), Delft, The Netherlands, 2013.

[64] H.A. Simon, The structure of ill-structured problems, Artificial Intelligence Models of discovery (4) (1973) 181-201.

[65] R. Snooks, Encoding Behavioral Matter, in: Y. Ikeda (Ed.), International Symposium on Algorithmic Design for Architecture and Urban Design (ALGODE), Vol. 1, Tokyo, Japan, 2011, p. 10.

[66] L. Soibelman, F. Pena-Mora, Distributed multi-reasoning mechanism to support conceptual structural design, Journal of Structural Engineering 126 (6) (2000) 733-742.

[67] S. Sugihara, Comparison between Top-Down and Bottom-Up Algorithms in Computational Design Practice, International Symposium on Algorithmic Design for Architecture and Urban Design, ALGODE Tokyo, 2011.

[68] S. Sugihara, iGeo: Algorithm Development Environment for Computational Design Coders with Integration of NURBS Geometry Modeling and Agent Based Modeling, in: D.J. Gerber, J. Sanchez, A. Huang (Eds.), ACADIA 14: Design Agency, Vol. 1, eVolo, Los Angeles, 2014, pp. 23-32.

[69] K. Sycara, Multiagent systems, AI magazine 19 (2) (1998) 79-92.

[70] M. Tambe, Implementing agent teams in dynamic multiagent environments, Applied Artificial Intelligence 12 (2-3) (1998) 189-210.

[71] M. Tambe, Teamwork in real-world, dynamic environments, University of Southern California, Information Sciences Institute, 1996. 
[72] M. Tambe, Towards flexible teamwork, Journal of artificial intelligence research (1997) 83-124.

[73] K. Terzidis, Algorithmic architecture, Elsevier, Architectural Press, Burlington,MA, 2006.

[74] M. Tsiliakos, Swarm Materiality: A multi-agent approach to stress driven material organization, Digital Physicality - Proceedings of the 30th eCAADe Conference, Vol. 1, 2012, pp. 301-309.

[75] G. Weiss, Multiagent Systems: A Modern Approach to Distributed Artificial Intelligence, (1999) 619.

[76] J. Werfel, Y. Bar-Yam, D. Rus, R. Nagpal, Distributed Construction by Mobile Robots with Enhanced Building Blocks, Complex Systems Institute, New England, 2006.

[77] J. Werfel, K. Petersen, R. Nagpal, Designing Collective Behavior in a TermiteInspired Robot Construction Team, Science 343 (2014) 754-758.

[78] M. Woodridge, N.R. Jennings, Intelligent Agents: Theory and Practice, Knowledge Engineering Review 10 (1995) 115-152.

[79] A. Yezioro, D. Bing, F. Leite, An applied artificial intelligence approach towards assessing building performance simulation tools, Energy and Buildings 40 (4) (2008) 612-620.

[80] Y.K. Yi, A.M. Malkawi, Optimizing building form for energy performance based on hierarchical geometry relation, Automation in Construction 18 (6) (2009) 825833. 